\title{
Amelanotic Irido-Ciliary Ring Melanoma: A Clinicopathological Correlation
}

\author{
Hassan A. Aziz ${ }^{a}$ Yasha S. Modi ${ }^{a}$ Thomas P. Plesec ${ }^{b}$ Arun D. Singh ${ }^{a}$ \\ ${ }^{a}$ Department of Ophthalmology, Cole Eye Institute, and ${ }^{b}$ Department of Pathology, Robert J. Tomsich Pathology \\ and Laboratory Institute, Cleveland Clinic Foundation, Cleveland, Ohio, USA
}

\section{Key Words}

Iris - Melanoma $\cdot$ Amelanotic irido-ciliary ring melanoma

\begin{abstract}
Purpose: To report a case of an amelanotic irido-ciliary ring melanoma. Design: Interventional case report. Results: A 44-year-old male was followed for asymptomatic amelanotic iris nevus of the right eye that was noted to have a localized ciliary body mass with ring extension along the trabecular meshwork. Fine needle aspiration biopsy was consistent with malignant melanoma. The patient underwent enucleation and remains disease free at 9 years of follow-up. Histopathology revealed malignant melanoma involving the iris and ciliary body with a 360-degree extension along the trabecular meshwork. The tumor was composed of a mixture of spindled and epithelioid cells with scant pigmentation. Conclusions: Amelanotic irido-ciliary ring melanoma with growth along the trabecular meshwork is a rare form of uveal melanoma that could present as an inconspicuous amelanotic iris mass.
\end{abstract}

(c) 2015 S. Karger AG, Basel

\section{Introduction}

Ring melanomas of the iris and ciliary body are rare and represent only $0.3 \%$ of all uveal melanomas [1]. The diagnosis is challenging as it typically presents with inconspicuous and nonspecific clinical and diagnostic fea- tures [1]. Careful examination, ancillary testing and a high degree of suspicion are needed to establish the diagnosis. Although pigmentation generally helps in establishing the diagnosis of uveal melanoma, up to one fourth of uveal melanomas are amelanotic.

To our knowledge, this is the first reported case in the English literature of an amelanotic irido-ciliary ring melanoma with growth along the trabecular meshwork. Herein, we describe the clinical features, evaluation and management of this case.

\section{Case Report}

A 44-year-old male without significant past medical history was referred to the ocular oncology service at the Cole Eye Institute for evaluation of an inconspicuous iris nevus of the right eye known to be present for about 5 years.

On presentation, his visual acuity was $20 / 20$ in both eyes, and the intraocular pressures were 17 and $15 \mathrm{~mm} \mathrm{Hg}$ in the right eye and left eye, respectively. Irides were blue in color with no apparent heterochromia or melanocytosis. Examination of his anterior and posterior segment of the left eye was normal.

Anterior examination of the right eye revealed an amelanotic multinodular iris mass that involved the anterior chamber angle from 7:30 to 9 o'clock with associated fine intrinsic vessels (fig. 1a). On gonioscopy, the amelanotic lesion extended from 10:30 to 7:30 (270 degrees) along the trabecular meshwork with associated inferior seeding (fig. $1 \mathrm{~b}, \mathrm{c}$ ). The lens was clear, and the posterior segment exam was normal. Ultrasound biomicroscopy (UBM) revealed only a localized ciliary body enlargement from 7 to 8 o'clock, with a maximum thickness of $2 \mathrm{~mm}$ (fig. 2).

\section{KARGER}

E-Mail karger@karger.com

www.karger.com/oop (c) 2015 S. Karger AG, Basel

2296-4681/15/0023-0153\$39.50/0
Arun D. Singh, MD

Department of Ophthalmic Oncology

Cole Eye Institute, Cleveland Clinic

2022 E 105th St, Cleveland, OH 44106 (USA)

E-Mail singha@ccf.org 

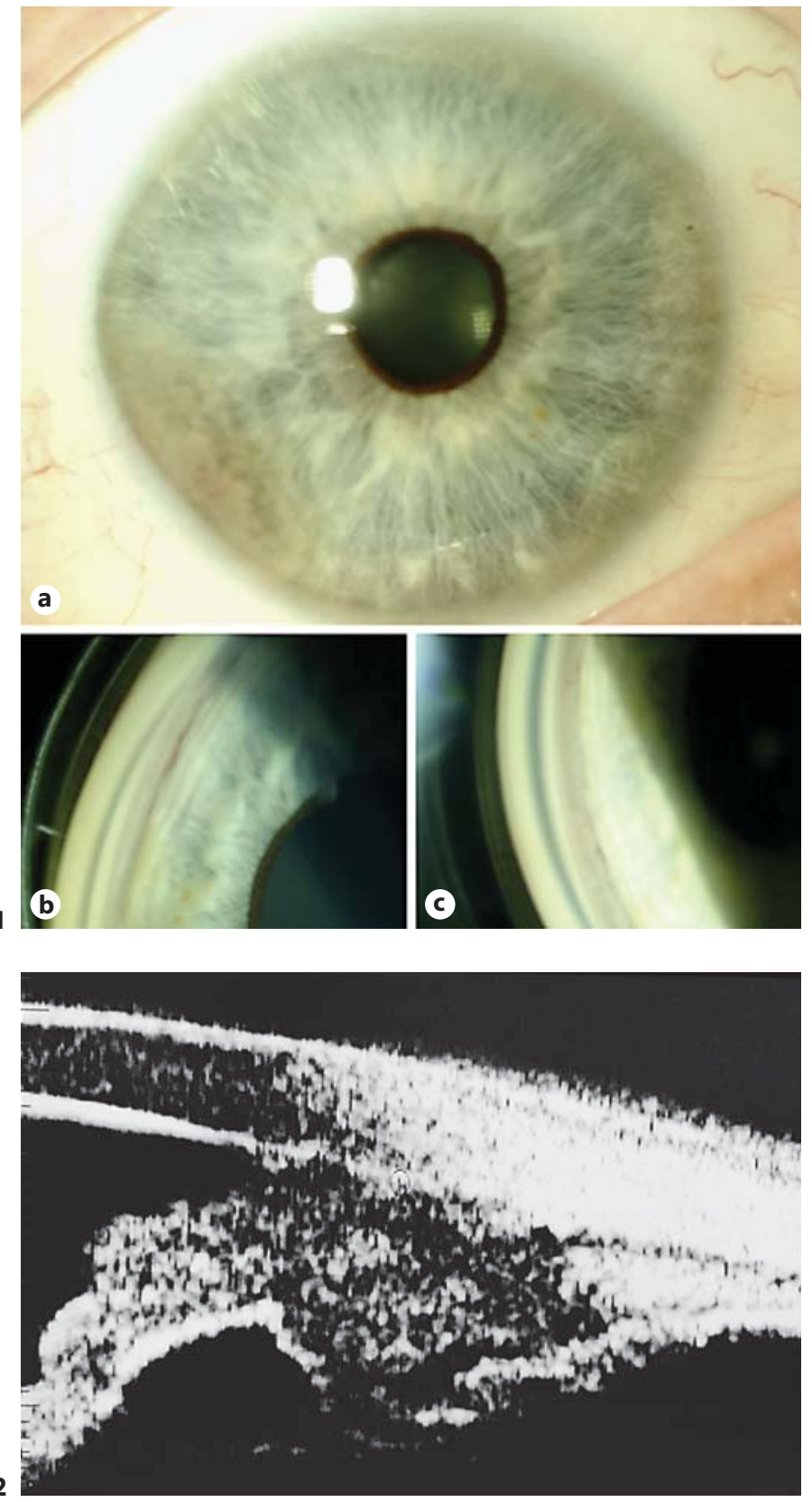

Fig. 1. a Slit-lamp photo of the right eye revealing an amelanotic multinodular iris mass from 7:30 to 9 o'clock with associated fine intrinsic vessels. $\mathbf{b}$ Gonioscopy photo of the angle showing extension of the amelanotic lesion from 3 to 5 o'clock. c Gonioscopy photo of the angle showing extension of the amelanotic lesion from 1 to 3 o'clock. Fig. 2. UBM of the right eye revealing a localized ciliary body lesion from 7 to 8 o'clock, with a maximum thickness of $2 \mathrm{~mm}$.
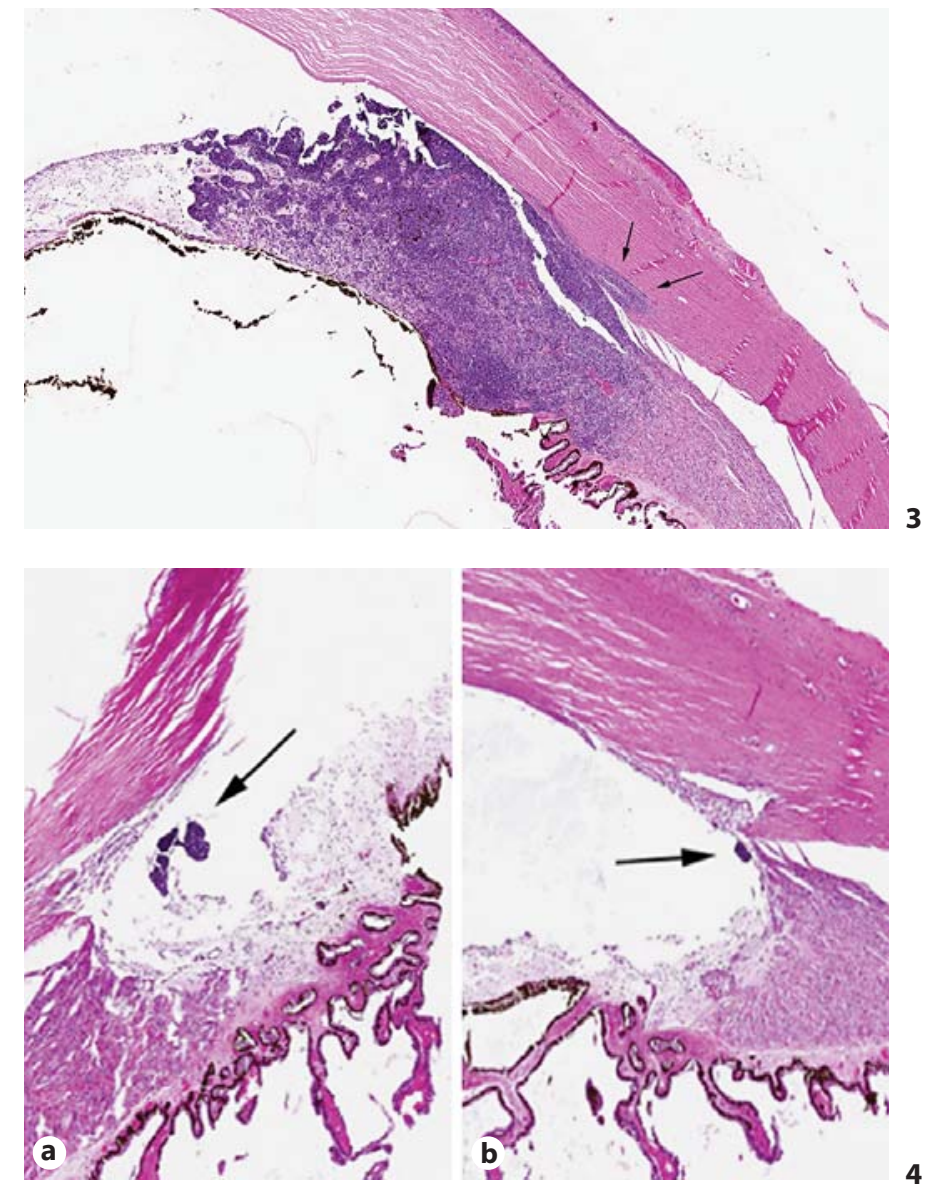

4

Fig. 3. Hematoxylin and eosin-stained section (40× magnification) of the main tumor mass. The melanoma is centered on the iris with extension into the ciliary body and trabecular meshwork (arrows). Fig. 4. Hematoxylin and eosin-stained section ( $24 \times$ magnification) of the peripheral calotte. Microscopic tumor fragments are seen adherent to the angles 180 degrees apart (arrows in $\mathbf{a}$ and $\mathbf{b}$ ), consistent with the clinical impression of ring-like extension.

The differential diagnosis at this point included: amelanotic melanoma, lymphoma, metastatic disease and inflammatory or infectious granuloma. The patient underwent fine needle aspiration biopsy (FNAB) of the ciliary body mass that was positive for HMB45-positive malignant melanoma cells. He subsequently underwent enucleation. Gross examination of the enucleation specimen revealed a thickened, tan-yellow area involving approximately three fourths of the iris circumference. Microscopically, the largest portion of the tumor was centered on the peripheral iris with extension into the ciliary body and trabecular meshwork (fig. 3). It was composed of a densely cellular mixture of epithelioid (about $25 \%$ ) and spindle cells (about 75\%). Mitotic figures were not identified in 40 high power fields, and melanin pigment was scant. In addition, nests of melanoma cells were seen in both angles in the medial calotte section (fig. 4).

On 9 years of follow-up, the patient continues to be symptom free with no evidence of systemic disease or ocular recurrence. 


\section{Discussion}

This case illustrates the presentation of a rare form of uveal melanoma. The amelanotic nature of the tumor made the diagnosis challenging, and FNAB was necessary to establish the diagnosis prior to enucleation. Of note, FNAB of iris lesions is a relatively safe procedure with low risk of complications or tumor seeding.

Ring melanomas involving the trabecular meshwork and ciliary body are rare, and the literature is restricted to a few case reports and small case series. To our knowledge, this is the first case of an amelanotic irido-ciliary melanoma with growth along the trabecular meshwork. Ciliary body melanoma portends a poor prognosis with a metastasis rate of up to $50 \%$, likely secondary to a delayed detection and subsequent diagnosis [1]. Lee et al. [2] reported on two cases of amelanotic irido-ciliary ring melanoma with delayed diagnosis; one was in a 23 -year-old man who was treated for 2 years for unilateral glaucoma, and diagnosis was only established after development of overt metastasis.

In the present case, the patient was diagnosed with an irido-ciliary melanoma with spread along the trabecular meshwork after being referred for evaluation of an asymptomatic amelanotic iris nevus. Although gonioscopy indicated a 270-degree ring-like extension, the ring-like growth could not be confirmed with certainty on UBM, which only showed ciliary body extension. Therefore, FNAB was necessary to confirm the diagnosis of amelanotic melanoma.
Treatment options for circumferential melanoma of the ciliary body and angle structures include enucleation or proton beam radiation. Gragoudas et al. [3] reported a recurrence rate of up to $67 \%$ after proton beam radiation. Demirci et al. [1] reported enucleation in all 23 patients (100\%) in the largest reported series of ring melanomas of the ciliary body. Despite enucleation, there was a metastasis rate of $52 \%$. Our patient underwent enucleation and remains disease free after 9 years of follow-up. He continues to undergo annual systemic surveillance with hepatic ultrasound because of risk of late metastasis [4].

Amelanotic irido-ciliary melanoma with ring extension along the trabecular meshwork is a rare form of uveal melanoma that may present as an inconspicuous amelanotic iris mass. In cases with a high index of suspicion based upon clinical findings, FNAB may be warranted to establish definitive diagnosis [5].

\section{Statement of Ethics}

The study protocol has been approved by the Institute's Committee on Human Research. The study application was approved as Exempt Research by the Institutional Review Board for the collection of data in an anonymous manner in which participants were not identifiable.

\section{Disclosure Statement}

The authors declare no conflicts of interest.

\section{References}

1 Demirci H, Shields CL, Shields JA, Honavar SG, Eagle RC Jr: Ring melanoma of the ciliary body: report on twenty-three patients. Retina 2002;22:698-706; quiz 852-853.

2 Lee V, Cree IA, Hungerford JL: Ring melanoma - a rare cause of refractory glaucoma. Br J Ophthalmol 1999;83:194-198.

3 Gragoudas ES, Lane AM, Munzenrider J, Egan KM, Li W: Long-term risk of local failure after proton therapy for choroidal/ciliary body melanoma. Trans Am Ophthalmol Soc 2002;100:43-48; discussion 48-49.
4 Schoenfield L: Uveal melanoma: a pathologist's perspective and review of translational developments. Adv Anat Pathol 2014;21:138143.

5 Singh AD, Pelayes DE, Brainard JA, Biscotti CV: History, indications, techniques and limitations. Monogr Clin Cytol 2012;21:1-9. 